\title{
A SEMICONTINUITY RESULT FOR MONODROMY UNDER DEGENERATION
}

\author{
NICHOLAS M. KATZ
}

\section{INTRODUCTION}

We fix a a prime number $l$. We denote by $E_{\lambda}$ a finite extension of $\mathbb{Q}_{l}$ inside a chosen algebraic closure $\overline{\mathbb{Q}}_{l}$ of $\mathbb{Q}_{l}$, by $\mathcal{O}_{\lambda}$ the ring of integers in $E_{\lambda}$, by $\mathbb{F}_{\lambda}$ its residue field, and by $\overline{\mathbb{F}}_{\lambda}$ an algebraic closure of $\mathbb{F}_{\lambda}$. We take as coefficient field $A$ one of the fields on the following list: $\mathbb{F}_{\lambda}, \overline{\mathbb{F}}_{\lambda}, E_{\lambda}$, or $\overline{\mathbb{Q}}_{l}$.

We work over a field $k$ in which $l$ is invertible. We are given a smooth connected $k$-scheme $S / k$, separated and of finite type, of dimension $r \geq 1$. In $S$, we are given a reduced and irreducible closed subscheme $Z$, of some dimension $d \geq 0$. We assume that an open dense set $V_{1} \subset Z$ is smooth over $\mathrm{k}$ (a condition which is automatic if the ground field $k$ is perfect).

On $S$, we are given a constructible $A$-sheaf $\mathcal{F}$. Because $\mathcal{F}$ is constructible, its restriction to $S-Z$ is constructible, so there exists a dense open set $U$ in $S-Z$ on which $\mathcal{F}$ is lisse. Similarly, the restriction of $\mathcal{F}$ to $V_{1}$ is lisse, so there exists a dense open set $V$ in $V_{1}$ on which $\mathcal{F}$ is lisse. Let us denote by $j$ the inclusion of $U$ into $S$, and by $i$ the inclusion of $V$ into $S$. Thus we have a lisse $A$-sheaf $j^{\star} \mathcal{F}$ on $U$, and a lisse $A$-sheaf $i^{\star} \mathcal{F}$ on $V$.

In this generality, there is absolutely nothing one can say relating the monodromy of the lisse $A$-sheaf $i^{\star} \mathcal{F}$ on $V$ to the monodomy of the lisse $A$-sheaf $j^{\star} \mathcal{F}$ on $U$. However, there is a class of constructible $A$-sheaves $\mathcal{F}$ on $S$ for which these monodromies are related, namely those "of perverse origin".

We say that a constructible $A$-sheaf $\mathcal{F}$ on $S$ is of perverse origin if there exists a perverse $A$-sheaf $M$ on $S$ such that

$$
\mathcal{F} \cong \mathcal{H}^{-r}(M)
$$

We say that any such perverse sheaf $M$ gives rise to $\mathcal{F}$.

The geometric interest of this notion is that, as we shall recall below (cf Corollaries 5 and 6 ), for any affine morphism $f: X \rightarrow S$, and for any perverse $A$-sheaf $M$ on $X$, the constructible $A$-sheaf

$$
R^{-r} f_{!} M:=\mathcal{H}^{-r}\left(R f_{!}(M)\right)
$$

Date: April 16, 2001. 
on $S$ is of perverse origin. In particular, suppose $X / k$ is a local complete intersection, everywhere of dimension $n+r$. Then for any lisse $A$-sheaf $\mathcal{G}$ on $X$,

$$
M:=\mathcal{G}[\operatorname{dim}(X)]=\mathcal{G}[r+n]
$$

is perverse on $X$. Hence for any affine morphism $f: X \rightarrow S$, the constructible A-sheaf

$$
R^{n} f_{1} \mathcal{G}=\mathcal{H}^{-r}\left(R f_{!}(M)\right)
$$

on $S$ is of perverse origin .

Our main result is that for $\mathcal{F}$ of perverse origin, the monodromy of the lisse $A$-sheaf $i^{\star} \mathcal{F}$ on $V$ is "smaller" than the monodromy of the lisse $A$-sheaf $j^{\star} \mathcal{F}$ on $U$.

To make this precise, let us pick geometric points $u$ of $U$ and $v$ of $V$. We have monodromy homomorphisms

$$
\rho_{U}: \pi_{1}(U, u) \rightarrow \operatorname{Aut}_{A}\left(\mathcal{F}_{u}\right)
$$

and

$$
\rho_{V}: \pi_{1}(V, v) \rightarrow \operatorname{Aut}_{A}\left(\mathcal{F}_{v}\right)
$$

attached to $j^{\star} \mathcal{F}$ on $U$ and to $i^{\star} \mathcal{F}$ on $V$ respectively. We define compact subgroups

$$
\Gamma_{U}:=\operatorname{Image}\left(\rho_{U}\right) \subset \operatorname{Aut}_{A}\left(\mathcal{F}_{u}\right)
$$

and

$$
\Gamma_{V}:=\operatorname{Image}\left(\rho_{V}\right) \subset A u t_{A}\left(\mathcal{F}_{v}\right)
$$

Theorem 1. For $S / k$ smooth and connected of dimension $r \geq 1$ and for $\mathcal{F}$ a constructible A-sheaf on $S$ of perverse origin, the group $\Gamma_{V}$ is isomorphic to a subquotient of the group $\Gamma_{U}$. More precisely, there exists a compact group D, a continuous group homomorphism

$$
D \rightarrow \Gamma_{U}
$$

a closed normal subgroup

$$
I \triangleleft D,
$$

and an A-linear embedding

$$
\mathcal{F}_{v} \subset \mathcal{F}_{u}^{I}
$$

with the following property: if we view $\mathcal{F}_{u}^{I}$ as a representation of $D / I$, then the subspace

$$
\mathcal{F}_{v} \subset \mathcal{F}_{u}^{I}
$$

is $D / I$-stable, and under the induced action of $D / I$ on $\mathcal{F}_{v}$, the image of $D / I$ in $\operatorname{Aut}_{A}\left(\mathcal{F}_{v}\right)$ is the group $\Gamma_{V}$.

Before giving the proof of the theorem, we must develop some basic properties of sheaves of perverse origin. 


\section{BASIC PROPERTIES OF SHEAVES OF PERVERSE ORIGIN}

Throughout this section, $S / k$ is smooth and connected, separated and of finite type, of dimension $r \geq 1$, and $\mathcal{F}$ is a constructible $A$-sheaf on $S$ of perverse origin.

Recall that for $M$ a perverse $A$-sheaf on $S$, its ordinary cohomology sheaves $\mathcal{H}^{i}(M)$ vanish for $\mathrm{i}$ outside the interval [-r, 0]. This is obvious for simple objects from their explicit description as middle extensions, and it follows for the general case because any perverse sheaf is a successive extension of finitely many simple objects, cf. [BBD, 2.1.11 and 4.3.1].

Recall that attached to any object $K$ in $D_{c}^{b}(S, A)$ are its perverse cohomology sheaves ${ }^{p} \mathcal{H}^{i}(K)$ : these are perverse $A$-sheaves on $S$, all but finitely many of which vanish. Their behavior under shifts is given by

$$
{ }^{p} \mathcal{H}^{i}(K[j])={ }^{p} \mathcal{H}^{i+j}(K)
$$

A distinguished triangle gives rise to a long exact sequence of perverse cohomology sheaves.

Given two integers $a \leq b$, an object $K$ in $D_{c}^{b}(S, A)$ is said to lie in ${ }^{p} D^{[a, b]}$ if its perverse cohomology sheaves ${ }^{p} \mathcal{H}^{i}(K)$ vanish for $i$ outside the closed interval $[a, b]$. Similarly, an object $K$ in $D_{c}^{b}(S, A)$ is said to lie in ${ }^{p} D^{\geq a}$ (respectively in ${ }^{p} D \leq b$ ) if its perverse cohomology sheaves ${ }^{p} \mathcal{H}^{i}(K)$ vanish for $i<a$ (respectively for $i>b$ ). An object of ${ }^{p} D^{[a, a]}$ is precisely an object of the form $M[-a]$ with $M$ perverse. For $a<b$, any object $K$ of ${ }^{p} D^{[a, b]}$ is a successive extension of its shifted perverse cohomology sheaves ${ }^{p} \mathcal{H}^{i}(K)[-i], i \in[a, b]$. More precisely, any object $K$ of ${ }^{p} D^{[a, b]}$ sits in a distinguished triangle

$$
{ }^{p} \mathcal{H}^{a}(K)[-a] \rightarrow K \rightarrow{ }^{p} \tau_{\geq a+1}(K) \rightarrow
$$

with the third term ${ }^{p} \tau_{\geq a+1}(K)$ in ${ }^{p} D^{[a+1, b]}$.

Lemma 2. Let $K$ be an object of ${ }^{p} D^{[a, b]}$. Then its ordinary cohomology sheaves $\mathcal{H}^{i}(K)$ vanish for $i$ outside the interval $[a-r, b]$.

Proof. We proceed by induction on $b-a$. If $K$ lies in ${ }^{p} D^{[a, a]}$, then $K$ is $M[-a]$ with $M$ perverse. The ordinary cohomology sheaves of $M, \mathcal{H}^{i}(M)$, vanish for $i$ outside the interval $[-r, 0]$. So those of $M[-a]$ vanish outside $[a-r, a]$. To do the induction step, use the distinguished triangle

$$
{ }^{p} \mathcal{H}^{a}(K)[-a] \rightarrow K \rightarrow{ }^{p} \tau_{\geq a+1}(K) \rightarrow
$$

above, and its long exact cohomology sequence of ordinary cohomology sheaves.

Corollary 3. Let $K$ be an object of ${ }^{p} D^{\geq a}$. Then its ordinary cohomology sheaves $\mathcal{H}^{i}(K)$ vanish for $i<a-r$.

Proposition 4. Let $K$ be an object of ${ }^{p} D^{\geq 0}$ on $S$. Then its $-r$ 'th ordinary cohomology sheaf $\mathcal{H}^{-r}(K)$ is of perverse origin. 
Proof. We have a distinguished triangle

$$
M \rightarrow K \rightarrow{ }^{p} \tau_{\geq 1}(K) \rightarrow
$$

whose first term $M:={ }^{p} \mathcal{H}^{0}(K)$ is perverse, and whose last term ${ }^{p} \tau_{\geq 1}(K)$ lies in ${ }^{p} D^{\geq 1}$. From the long exact cohomology sequence for ordinary cohomology sheaves, we find

$$
\mathcal{H}^{-r}(M) \cong \mathcal{H}^{-r}(K) \text {. }
$$

Corollary 5. For any affine morphism $f: X \rightarrow S$, and for any perverse $A$-sheaf $M$ on $X$, the constructible $A$-sheaf

$$
R^{-r} f_{!} M:=\mathcal{H}^{-r}\left(R f_{!}(M)\right)
$$

on $S$ is of perverse origin.

Proof. Indeed, one knows [BBD, 4.1.1] that for an affine morphism $f, R f_{\star}$ maps ${ }^{p} D \leq 0$ on $X$ to ${ }^{p} D^{\leq 0}$ on $S$. Dually, $R f_{!}$maps ${ }^{p} D^{\geq 0}$ on $X$ to ${ }^{p} D^{\geq 0}$ on $S$ [BBD, 4.1.2]. So for $M$ perverse on $X, R f_{!} M$ lies in ${ }^{p} D^{\geq 0}$ on $S$, and we apply to it the previous result.

Corollary 6. Suppose $X / k$ is a local complete intersection, everywhere of dimension $n+r$. For any lisse $A$-sheaf $\mathcal{G}$ on $X$, and any affine morphism $f: X \rightarrow S$, the constructible $A$-sheaf $R^{n} f_{1} \mathcal{G}$ on $S$ is of perverse origin .

Proof. Because $X / k$ is a local complete intersection, everywhere of dimension $n+r$, given any lisse $A$-sheaf $\mathcal{G}$ on $X$, the object

$$
M:=\mathcal{G}[\operatorname{dim}(X)]=\mathcal{G}[r+n]
$$

is perverse on $X$. [See [Ka-PES II, Lemma 2.1] for the case when $\mathcal{G}$ is the constant sheaf $A$, and reduce to this case by observing that if $K$ is perverse on $X$ and $\mathcal{G}$ is lisse on $X$, then $\mathcal{G} \otimes_{A} K$ is perverse on $X$.] Now apply the previous result to $M$.

Proposition 7. Let $\mathcal{F}$ be of perverse origin on $S$. For any connected smooth $k$ scheme $T / k$, and for any $k$-morphism $f: T \rightarrow S$, the pullback $f^{\star} \mathcal{F}$ is of perverse origin on $T$.

Proof. We factor $f$ as the closed immersion $i$ of $T$ into $T \times_{k} S$ by ( $\left.i d, f\right)$, followed by the projection $p_{2}$ of $T \times_{k} S$ onto $S$. So it suffices to treat separately the case when $f$ is smooth, everywhere of some relative dimension $a$, and the case when $f$ is a regular closed immersion, everywhere of some codimension $b$. Pick $M$ perverse on $S$ giving rise to $\mathcal{F}$. In the first case, $K:=f^{\star} M[a]$ is perverse on $T$ [BBD, paragraph above 4.2.5], $\operatorname{dim}(T)=r+a$, and

$$
f^{\star} \mathcal{F}=f^{\star} \mathcal{H}^{-r}(M)=\mathcal{H}^{-r-a}\left(f^{\star} M[a]\right)=\mathcal{H}^{-r-a}(K)
$$

is thus of perverse origin on $T$. In the second case, $K:=f^{\star} M[-b]$ lies in ${ }^{p} D^{[0, b]}$ (apply [BBD, 4.1.10(ii)] b times Zariski locally on $T$, and observe that the property 
of lying in ${ }^{p} D^{[0, b]}$ can be checked Zariski locally, since it amounts to the vanishing of certain perverse cohomology sheaves), $\operatorname{dim}(T)=r-b$, and

$$
f^{\star} \mathcal{F}=f^{\star} \mathcal{H}^{-r}(M)=\mathcal{H}^{b-r}\left(f^{\star} M[-b]\right)=\mathcal{H}^{b-r}(K)
$$

is thus, by the previous Proposition, of perverse origin on $T$.

Proposition 8. Let $\mathcal{F}$ be of perverse origin on $S$. Given a connected smooth $k$ scheme $T / k$ of dimension a, with function field $k(T)$ and generic point $\eta:=\operatorname{Spec}(k(T))$, and given a smooth $k$-morphism $f: S \rightarrow T$ with generic fibre $S_{\eta} / k(T)$, the restriction $\mathcal{F}_{\eta}:=\mathcal{F} \mid S_{\eta}$ is of perverse origin on $S_{\eta} / k(T)$.

Proof. Indeed, for $M$ perverse on $S$ giving rise to $\mathcal{F}, M_{\eta}[-a]$ is perverse on $S_{\eta} / k(T)$ and gives rise to $\mathcal{F}_{\eta}$.

Proposition 9. Let $\mathcal{F}$ be of perverse origin on $S$. For $j: U \rightarrow S$ the inclusion of any dense open set on which $\mathcal{F}$ is lisse, the canonical map

$$
\mathcal{F} \rightarrow j_{\star} j^{\star} \mathcal{F}
$$

is injective.

Proof. Let $M$ be a perverse $A$-sheaf on $S$ which gives rise to $\mathcal{F}$. We know [BBD, 4.3.1] that the category of perverse $A$-sheaves on $S$ is an abelian category which is both artinian and noetherian, so every object is a successive extension of finitely many simple objects. We proceed by induction on the length of $M$.

If $M$ is simple, then in fact we have $\mathcal{F} \cong j_{\star} j^{\star} \mathcal{F}$. To see this, we distinguish two cases. The first case is that $M$ is supported in an irreducible closed subscheme $W$ of $S$ with $\operatorname{dim}(W) \leq r-1$. In this case its ordinary cohomology sheaves $\mathcal{H}^{i}(M)$ vanish for $i$ outside the closed interval $[1-r, 0]$. Thus $\mathcal{F}=0$ in this case, so the assertion trivially holds. The second case is that $M$ is the middle extension of its restriction to any dense open set on which it is lisse. In this case $M$ is $j_{\star !}\left(j^{\star} \mathcal{F}[r]\right)$, and from the explicit description [BBD, 2.1.11] of middle extension we see that

$$
\mathcal{H}^{-r}(M)=j_{\star} j^{\star} \mathcal{F} \text {. }
$$

In the general case, we pick a simple subobject $M_{1}$ of $M$, and denote

$$
M_{2}:=M / M_{1} \text {. }
$$

We put

$$
\mathcal{F}_{i}:=\mathcal{H}^{-r}\left(M_{i}\right)
$$

for $i=1,2$. Then the short exact sequence

$$
0 \rightarrow M_{1} \rightarrow M \rightarrow M_{2} \rightarrow 0
$$

leads to a left exact sequence

$$
0 \rightarrow \mathcal{F}_{1} \rightarrow \mathcal{F} \rightarrow \mathcal{F}_{2}
$$


By induction, we know that

$$
\mathcal{F}_{i} \hookrightarrow j_{\star} j^{\star} \mathcal{F}_{i}
$$

for $i=1,2$. A simple diagram chase shows that $\mathcal{F} \hookrightarrow j_{\star} j^{\star} \mathcal{F}$, as required.

\section{Proof of the theorem}

Proof. If the theorem is true for one choice of geometric points $u$ of $U$ and $v$ of $V$, it is true for any other choice. So we may assume that $u$ lies over the generic point of $U$, and that $v$ lies over the generic point of $V$. By Proposition 7, we may at will shrink $S$ to any dense open set $S^{\prime} \subset S$ which meets $Z$, then replace $U$ and $V$ by their intersections with $S^{\prime}$. This changes neither $\Gamma_{U}$ nor $\Gamma_{V}$.

Denote by $Z_{1} \subset Z$ the closed subset $Z-V$. Shrinking $S$ to $S-Z_{1}$ we reduce to the case when $Z$ is smooth in $S$, and $\mathcal{F}$ is lisse on $Z$. Pulling $\mathcal{F}$ back to the blowup of $S$ along $Z$, allowable by Proposition 7 , changes neither $\Gamma_{U}$ nor $\Gamma_{V}$, and reduces us to the case where $Z$ is a connected smooth divisor in $S$.

We now focus on the relative dimension $r$ of $S / k$. We first treat the case $r=1$. In this case, $S$ is a smooth connected curve over $k$, and $Z$ is a closed connected subscheme of $S$ which is etale over $k$. Thus $Z$ is a closed point $\operatorname{Spec}(L)$ of $S$, with $L / k$ a finite separable extension. Deleting from $S$ the finitely many closed points other than $Z$ at which $\mathcal{F}$ is not lisse, we may further assume that $\mathcal{F}$ is a sheaf of perverse origin on $S$ which is lisse on $S-Z$.

We now come to the essential point, that denoting by $j$ the inclusion $S-Z \subset S$, the canonical map

$$
\mathcal{F} \rightarrow j_{\star} j^{\star} \mathcal{F}
$$

is injective (by Proposition 9).

Let us spell out what this mean concretely (compare [Mil, II.3.12 and II.3.16]). Denote by $K$ the function field of $S$, by

$$
\eta: \operatorname{Spec}(K) \rightarrow S
$$

the (inclusion of the) generic point of $S$, by $\bar{K}$ an algebraic closure of $K$, by

$$
\bar{\eta}: \operatorname{Spec}(\bar{K}) \rightarrow S
$$

the (inclusion of the) corresponding geometric generic point of S, and by $K^{\text {sep }} \subset \bar{K}$ the separable closure of $K$ inside $\bar{K}$. The stalk $\mathcal{F}_{\bar{\eta}}$ is the representation of $\operatorname{Gal}\left(K^{\text {sep }} / K\right)$ obtained from viewing $\mathcal{F} \mid S-Z$ as a representation of $\pi_{1}(S-Z, \bar{\eta})$ and composing with the canonical surjection

$$
\operatorname{Gal}\left(K^{\text {sep }} / K\right) \rightarrow \pi_{1}(S-Z, \bar{\eta}) .
$$

View the closed point $Z$ as a discrete valuation $v$ of $K$, and extend $v$ to a valuation $\bar{v}$ of $\bar{K}$, with valuation ring $\mathcal{O}_{\bar{v}} \subset \bar{K}$. The residue field of $\mathcal{O}_{\bar{v}}$ is an algebraic closure of $L$, so a geometric generic point $\bar{z}$ of $Z$. Inside $\operatorname{Gal}\left(K^{\operatorname{sep}} / K\right)$, we have the corresponding 
decomposition group $D:=D_{\bar{v}}$, which contains as a normal subgroup the inertia group $I:=I_{\bar{v}}$. We have a short exact sequence of groups

$$
0 \rightarrow I \rightarrow D \rightarrow \operatorname{Gal}\left(L^{\text {sep }} / L\right) \rightarrow 0 .
$$

The stalk $\mathcal{F}_{\bar{z}}$ of $\mathcal{F}$ at $\bar{z}$ is the representation of $D / I \cong \operatorname{Gal}\left(L^{\text {sep }} / L\right) \cong \pi_{1}(Z, \bar{z})$ given by $\mathcal{F} \mid Z$. The stalk $\left(j_{\star} j^{\star} \mathcal{F}\right)_{\bar{z}}$ of $j_{\star} j^{\star} \mathcal{F}$ at $\bar{z}$ is the representation of $D / I \cong \operatorname{Gal}\left(L^{\text {sep }} / L\right) \cong$ $\pi_{1}(Z, \bar{z})$ on the subspace of $I$-invariants in the restriction to $D$ of the representation of $\operatorname{Gal}\left(K^{\text {sep }} / K\right)$ on $\mathcal{F}_{\bar{\eta}}$ :

$$
\left(j_{\star} j^{\star} \mathcal{F}\right)_{\bar{z}} \cong\left(\mathcal{F}_{\bar{\eta}}\right)^{I}
$$

For $\mathcal{F}$ any constructible sheaf on $S$ which is lisse on $S-Z$, the injectivity of

$$
\mathcal{F} \hookrightarrow j_{\star} j^{\star} \mathcal{F}
$$

means precisely that at the single point $Z$ we have an $A$-linear $D$-equivariant inclusion

$$
\mathcal{F}_{\bar{z}} \hookrightarrow\left(j_{\star} j^{\star} \mathcal{F}\right)_{\bar{z}} \cong\left(\mathcal{F}_{\bar{\eta}}\right)^{I}
$$

So to conclude the proof of the theorem in the case $r=1$, we have only to take $I \triangleleft D$ as our groups, and use the composite homomorphism

$$
D \hookrightarrow \operatorname{Gal}\left(K^{\text {sep }} / K\right) \rightarrow \pi_{1}(S-Z, \bar{\eta}) \rightarrow \operatorname{Aut}_{A}\left(\mathcal{F}_{\bar{\eta}}\right)
$$

to map $D$ to

$$
\Gamma_{U}:=\operatorname{Image}\left(\pi_{1}(S-Z, \bar{\eta}) \rightarrow \operatorname{Aut}_{A}\left(\mathcal{F}_{\bar{\eta}}\right)\right)
$$

For general $r \geq 1$, we argue as follows. Recall that $\mathcal{F}$ is lisse on an open dense set $U \subset S-Z$, and is lisse on $Z$. Pick a closed point $z$ in $Z$. Shrinking $S$ to a Zariski open neighborhood of $z$ in $S$, we reduce to the case where there exist $r$ functions $s_{i}, i=1, \ldots, r$ on $S$ which define an etale $k$-morphism $S \rightarrow \mathbb{A}_{k}^{r}$ and such that $Z$ is defined in $S$ by the single equation $s_{r}=0$. Then the map $S \rightarrow \mathbb{A}_{k}^{r-1}$ defined by $s_{i}, i=1, \ldots, r-1$ is smooth of relative dimension 1 , and makes $Z$ etale over $\mathbb{A}_{k}^{r-1}$. Denote by $\mathrm{F}$ the function field of $\mathbb{A}_{k}^{r-1}$, and make the base change of our situation $(S, Z) / \mathbb{A}_{k}^{r-1}$ from $\mathbb{A}_{k}^{r-1}$ to $\operatorname{Spec}(F)$. This is allowable by Proposition 8. We obtain a situation $\left(S_{F}, Z_{F}\right) / F$ in which $S_{F}$ is a connected smooth curve over $F$, and $Z_{F}$ is a nonvoid connected closed subscheme of $S_{F}$ which is etale over $F$. Thus $Z_{F}$ is a closed point $\operatorname{Spec}(L)$ of $S_{F}$, with $L$ a finite separable extension of $F$. The $r=1$ case of the theorem applies to this situation over $F$. Its truth here gives the theorem for our situation $(S, Z) / \mathbb{A}_{k}^{r-1}$. Indeed, the connected normal schemes $U$ and $U_{F}$ have the same function fields, and, being normal, their fundamental groups are both quotients of the absolute galois group of their common function field. So the groups $\Gamma_{U}$ and $\Gamma_{U_{F}}$ coincide. Similarly for $V=Z$, the groups $\Gamma_{V}$ and $\Gamma_{V_{F}}$ coincide. 


\section{APPlication to Zariski closures of MONODROMY gROUPS}

As an immediate corollary of the theorem, we obtain:

Corollary 10. Hypotheses and notations as in the theorem, denote by $N_{U}$ the rank of the lisse $A$-sheaf $j^{\star} \mathcal{F}$ on $U$, and by $N_{V}$ the rank of the lisse $A$-sheaf $i^{\star} \mathcal{F}$ on $V$.

(1) We have the inequality of ranks

$$
N_{V} \leq N_{U}
$$

(2) Suppose in addition that $A$ is $\overline{\mathbb{Q}}_{l}$. Denote by $G_{U}$ the $\overline{\mathbb{Q}}_{l}$-algebraic group which is the Zariski closure of $\Gamma_{U}$ in $A u t_{\overline{\mathbb{Q}}_{l}}\left(\mathcal{F}_{u}\right) \cong G L\left(N_{U}, \overline{\mathbb{Q}}_{l}\right)$, and denote by $G_{V}$ the $\overline{\mathbb{Q}}_{l}$ algebraic group which is the Zariski closure of $\Gamma_{V}$ in $A u t_{\overline{\mathbb{Q}}_{l}}\left(\mathcal{F}_{v}\right) \cong G L\left(N_{V}, \overline{\mathbb{Q}}_{l}\right)$. Then the algebraic group $G_{V}$ is a subquotient of $G_{U}$.

In particular, we have

(2a) if $G_{U}$ is finite (or equivalently if $\Gamma_{U}$ is finite) then $G_{V}$ is finite (or equivalently $\Gamma_{V}$ is finite),

(2b) $\operatorname{dim}\left(G_{V}\right) \leq \operatorname{dim}\left(G_{U}\right)$,

(2c) $\operatorname{rank}\left(G_{V}\right) \leq \operatorname{rank}\left(G_{U}\right)$.

\section{APPENDIX: WHEN AND WHERE IS A SHEAF OF PERVERSE ORIGIN LISSE?}

Proposition 11. Hypotheses and notations as in the theorem, the sheaf $\mathcal{F}$ of perverse origin on $S$ is lisse, say of rank $N$, if and only if its stalks $\mathcal{F}_{s}$ at all geometric points $s$ of $S$ have constant rank $N$.

Proof. It is trivial that if $\mathcal{F}$ is lisse on $S$, then its stalks have constant rank. Suppose now that $\mathcal{F}$ on $S$ is of perverse origin, and that all its stalks have constant $\operatorname{rank} N$. We must show that $\mathcal{F}$ is lisse on $S$.

It suffices to show that $\mathcal{F}$ is lisse on an open set $V \subset S$ whose complement $S-V$ has codimension $\geq 2$ in $S$. Indeed, by Zariski-Nagata purity, if we denote by $j: V \rightarrow S$ the inclusion, the lisse sheaf $j^{\star} \mathcal{F}$ on $V$ extends uniquely to a lisse sheaf $\mathcal{E}$ on $S$. For any lisse sheaf $\mathcal{E}$ on $S$, and any dense open set $V \subset S$, we have $\mathcal{E} \cong j_{\star} j^{\star} \mathcal{E}$. But $j^{\star} \mathcal{E} \cong j^{\star} \mathcal{F}$, so we find that $\mathcal{E} \cong j_{\star} j^{\star} \mathcal{F}$. In particular, $j_{\star} j^{\star} \mathcal{F}$ is lisse on $S$, and hence all its stalks have constant rank $N$. The injective (by Proposition 9) map

$$
\mathcal{F} \hookrightarrow j_{\star} j^{\star} \mathcal{F}
$$

must be an isomorphism, because at each geometric point the stalks of both source and target have rank $N$. Thus we find

$$
\mathcal{F} \cong j_{\star} j^{\star} \mathcal{F} \cong \mathcal{E}
$$

which shows that $\mathcal{F}$ is lisse on $S$.

We now show that an $\mathcal{F}$ of perverse origin on $S$ which has constant rank $N$ must be lisse. Thanks to the above discussion, we may remove from $S$ any closed set of codimension 2 or more. Thus we may assume that $\mathcal{F}$ is lisse on an open set 
$U \subset S$, inclusion denoted $j: U \rightarrow S$, and that that the complement $S-U$ is a disjoint union of finitely many irreducible divisors $Z_{i}$. Denote by $\eta$ the generic point of $S$. At the generic point $z_{i}$ of $Z_{i}$, the local ring $\mathcal{O}_{S, z_{i}}$ is a discrete valuation ring. For suitable geometric points $\bar{\eta}$ and $\bar{z}_{i}$ lying over $\eta$ and $z_{i}$ respectively, we have the inertia and decomposition groups $I_{i}$ and $D_{i}$. We have an injective (by Propostion 9) $D_{i}$-equivariant map

$$
\mathcal{F}_{\overline{z_{i}}} \hookrightarrow\left(j_{\star} j^{\star} \mathcal{F}\right)_{\overline{z_{i}}} \cong\left(\mathcal{F}_{\bar{\eta}}\right)^{I_{i}} \subset \mathcal{F}_{\bar{\eta}}
$$

As both $\mathcal{F}_{\overline{z_{i}}}$ and $\mathcal{F}_{\bar{\eta}}$ have the same rank $N$, all the displayed maps must be isomorphisms. Therefore $I_{i}$ acts trivially on $\mathcal{F}_{\bar{\eta}}$. Thus $j^{\star} \mathcal{F}$ is a lisse sheaf on $U$ which is unramified at the generic point of each $Z_{i}$. So by Zariski-Nagata purity, $j^{\star} \mathcal{F}$ extends to a lisse sheaf $\mathcal{E}$ on $S$. Exactly as in the paragraph above, we see that

$$
\mathcal{F} \cong j_{\star} j^{\star} \mathcal{F} \cong \mathcal{E}
$$

which shows that $\mathcal{F}$ is lisse on $S$.

Proposition 12. Hypotheses and notations as in the theorem, let $\mathcal{F}$ be of perverse origin on $S$. The integer-valued function on $S$ given by

$$
s \mapsto \operatorname{rank}\left(\mathcal{F}_{s}\right)
$$

is lower semicontinuous, i.e., for every integer $r \geq 0$, there exists a reduced closed subscheme $S_{<r} \subset S$ such that a geometric point $s$ of $S$ lies in $S_{<r}$ if and only if the stalk $\mathcal{F}_{s}$ has rank $\leq r$. If we denote by $N$ the generic rank of $\mathcal{F}$, then $S=S_{\leq N}$, and $S-S_{\leq N-1}$ is the largest open set on which $\mathcal{F}$ is lisse.

Proof. Once we show the lower semicontinuity of the rank, the second assertion is immediate from the preceeding proposition.

To show the lower semicontinuity, we first reduce to the case when $k$ is perfect. Indeed, for $k^{\text {per }}$ the perfection of $k$, and $S_{1}:=S \otimes_{k} k^{\text {per }}$, the natural map $\pi: S_{1} \rightarrow S$ is a universal homeomorphism, $S_{1} / k^{\text {per }}$ is smooth and connected, and $\pi^{\star} \mathcal{F}$ is of perverse origin on $S_{1} / k^{\text {per }}$. Thus it suffices to treat the case when $k$ is perfect.

Because $\mathcal{F}$ is a constructible sheaf, its rank function is constructible. So to show lower semicontinuity, it suffices to show that the rank decreases under specialization. Thus let $Z \subset S$ be an irreducible reduced closed subscheme, with geometric generic point $\bar{\eta}_{Z}$. We must show that at any geometric point $z \in Z$, we have

$$
\operatorname{rank}\left(\mathcal{F}_{\bar{\eta}_{Z}}\right) \leq \operatorname{rank}\left(\mathcal{F}_{z}\right)
$$

Because the field $k$ is perfect, we may, by de Jong [de Jong, Thm. 3.1], find a smooth connected $k$-scheme $Z_{1}$ and a proper surjective $k$-morphism $f: Z_{1} \rightarrow Z$. By Proposition $7, f^{\star} \mathcal{F}$ is of perverse origin on $Z_{1}$. By Corollary $10,(1)$, applied on $Z_{1}$, we get the asserted inequality of ranks.

Acknowledgement This work began as a diophantine proof of Parts (2a) and (2c) of the Corollary, in the special case when the ground field $k$ is finite, and when the 
sheaf $\mathcal{F}$ of perverse origin on $S$ is $R^{n} f ! \mathcal{G}$ for $f: X \rightarrow S$ a smooth affine morphism everywhere of relative dimension $\mathrm{n}$, with $\mathcal{G}$ a lisse sheaf on $X$. I owe to Deligne both the idea of formulating the theorem in terms of subquotients, and the idea that it applied to sheaves of perverse origin.

\section{REFERENCES}

[BBD] Beilinson, A. A., Bernstein, I. N., and Deligne, P., Faisceaux Pervers, Astérisque 100, 1982.

[de Jong] de Jong, A.J., Smoothness, semi-stability and alternations, Pub. Math. I.H.E.S. 83, 1996, 51-93.

[Ka-PES II] , Katz, N., Perversity and Exponential Sums II, pages 205-252 in Barsotti Symposium in Algebraic Geometry, (ed. Cristante and Messing), Academic Press, 1994.

[Ka-SE] Katz, N., Sommes Exponentielles, rédigé par G. Laumon, Astérisque 79, 1980.

[Mil] Milne, J.S., Étale Cohomology, Princeton University Press, 1980.

[SGA4] A. Grothendieck et al - Séminaire de Géométrie Algébrique du Bois-Marie 1963/64 SGA 4. Tome III, Springer Lecture Notes in Math. 305, 1973. 